\title{
Robust 3D Deformation Field Estimation by Template Propagation
}

\author{
P. Rösch ${ }^{1}$, T. Netsch ${ }^{1}$, M. Quist ${ }^{2}$, G.P. Penney ${ }^{3}$, D.L.G. Hill ${ }^{3}$, and J. Weese ${ }^{1}$ \\ 1 Philips Research Laboratories, Division Technical Systems, \\ Röntgenstraße 24-26, D-22335 Hamburg, Germany \\ 2 MIMIT Advanced Development, Philips Medical Systems Nederland B.V., \\ Veenpluis 4-6, NL-5680 DA Best, The Netherlands \\ 3 Radiological Sciences and Biomechanical Engineering. \\ The Guy's, King's and St Thomas' School of Medicine, \\ King's College London, London SE1 9RT, U.K.
}

\begin{abstract}
A new robust method to automatically determine a 3D motion vector field for medical images in the presence of large deformations is proposed. The central idea of this approach is template propagation. Starting from an image position where valid starting estimates are known, small sub-volumes (templates) are registered rigidly. Parameters of successfully registered templates serve as starting estimates for its neighbors. The registration proceeds layer by layer until the relevant image volume is covered. Based on this principle, a template-based registration algorithm has been implemented. Using the resulting set of corresponding points, the parameters of a non-rigid transformation scheme are determined. The complete procedure has been validated using four MR image pairs containing considerable deformations. In order to obtain an estimate for the accuracy, homologous points determined by template propagation are compared to corresponding landmarks defined by an expert. For landmarks with sufficient structure, the average deviation is well below the voxel size of the images. Because of the larger number of homologous points available, transformations incorporating the output of template propagation yielded a larger similarity between the reference image and the transformed image than an elastic transformation based on landmark pairs.
\end{abstract}

\section{Introduction}

For both medical and non-medical applications the generation of motion and deformation fields is a prerequisite for the automated analysis of image pairs. In medical applications it is desirable to distinguish between deformations caused by bending joints and moving organs on the one hand and pathologies on the other hand. Examples are following the size and shape of tumors, compensating respiratory motion in cardiac perfusion studies and comparison between preand post treatment. 

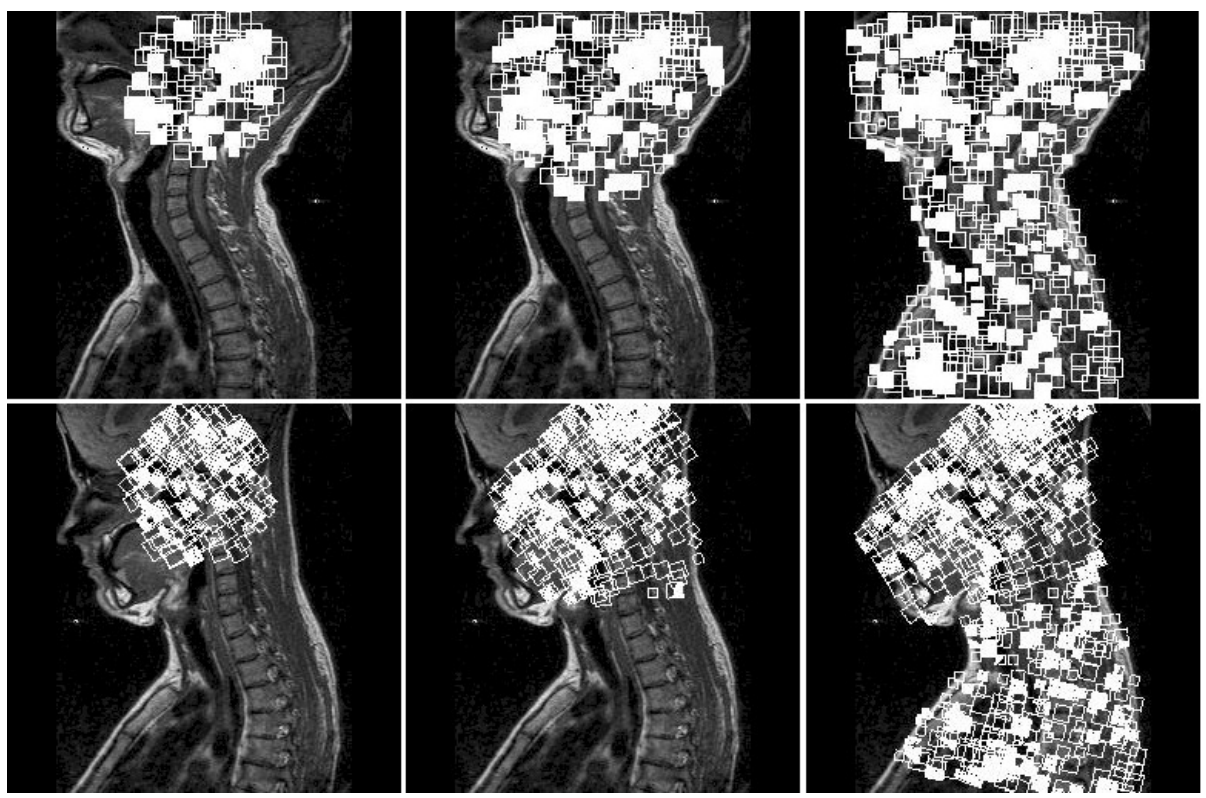

Fig.1 Process of 3D template propagation starting at one location where valid starting estimates are known (left) propagate (center) until the whole volume of overlap is covered with templates (right). The top row shows a slice of the reference image, the bottom row represents a slice of the target image.

One possibility for deformation field estimation is to select a set of corresponding landmarks manually and to determine the motion vectors at the other locations by interpolation [1]. The disadvantage of interpolation functions based on sparse data is that the properties of tissue are not taken into account and that the shapes of rigid bodies (e.g. bones) are affected by the transformation. In order to avoid this, the image can be segmented into parts that are deformed while other parts are kept rigid [2].

The methods described in the following attempt to reduce user interaction by automatically establishing correspondence between image locations. One class of automatic algorithms extract and match crest lines [3] or region boundaries [4]. The procedures yield transformation parameters for the selected feature lines only and the deformation field has to be extended to the whole volume afterwards.

Another method is iterative gray-value based elastic registration where the full image content is used. Motion field estimation is an optimization procedure aiming at the deformation field yielding maximum similarity between the images. Global optimization schemes for elastic registration change the whole deformation field during each refinement step. Thus, a regularization step e.g. by including biomechanical models [5] is essential to avoid local optima resulting from the large number of parameters. Another possibility is to vary a mesh of points 
controlling a free-form deformation and to introduce a penalty function to constrain the deformation to be smooth [6].

An alternative to global optimization is block-matching. The algorithm presented in [7] starts by registering the whole image rigidly. Afterwards the image is successively split into smaller and smaller portions which are again registered rigidly using the results of the "parent" block as starting estimates. Although this method works for images with relatively small deformations, the presence of large deformations in 3D image pairs (like fig.1) does not allow even for an approximate rigid matching of image parts that are larger than a few $\mathrm{cm}$ so that the initial step of this procedure can not be applied in the case of large deformations. The algorithm closest to the approach presented here is the one in [8] where displacement vectors at the nodes of a 3D grid are varied and the correlation of image features in the environment of the nodes is used as similarity measure. Registration starts at a coarse resolution and the resulting translation vectors are refined in subsequent steps at finer resolutions.

Rather than applying a multi-resolution strategy to determine local translation vectors, the procedure described in this contribution establishes a chain of successfully registered small sub-volumes (templates) from an image position with known translation and rotation parameters to the other locations. The volume of the templates is so small that the structures contained are not significantly affected by deformations. Thus, a rigid registration yields valid local translation and rotation parameters at the template center. In contrast to current block matching procedures, the template to be registered next is chosen based on the success of previous registration steps in its neighborhood. As a result, image regions that can not be "trespassed" by template propagation because they do not contain sufficient structure are circumvented. Apart from the propagation strategy, the choice of a similarity measure that is applicable to small volumes (e.g. $5 \times 5 \times 3$ voxels) is crucial for the success of this method. Two measures that fulfill these requirements, standard cross-correlation and local correlation (LC) 9 ] gave similar results for the example images. However, the applicability of crosscorrelation is limited to single modality registration, in the case of MR data it is even limited to images acquired with the same protocol. As LC has been successfully applied for single- and multi-modality registration [10] and future evaluations will include MR image pairs with different protocols and images originating from different modalities, only results based on LC are reported here.

The algorithm is described in section 2 Experimental results are given in section 3 and discussed in section 4 Finally, conclusions are drawn in section 5 .

\section{Algorithm}

This algorithm finds an elastic registration in three consecutive steps. First, templates are selected from the reference volume. Secondly, these templates are rigidly registered to the deformed volume by template propagation. Finally, an elastic transformation according to [2] based on the corresponding points originating from the previous step is performed. 

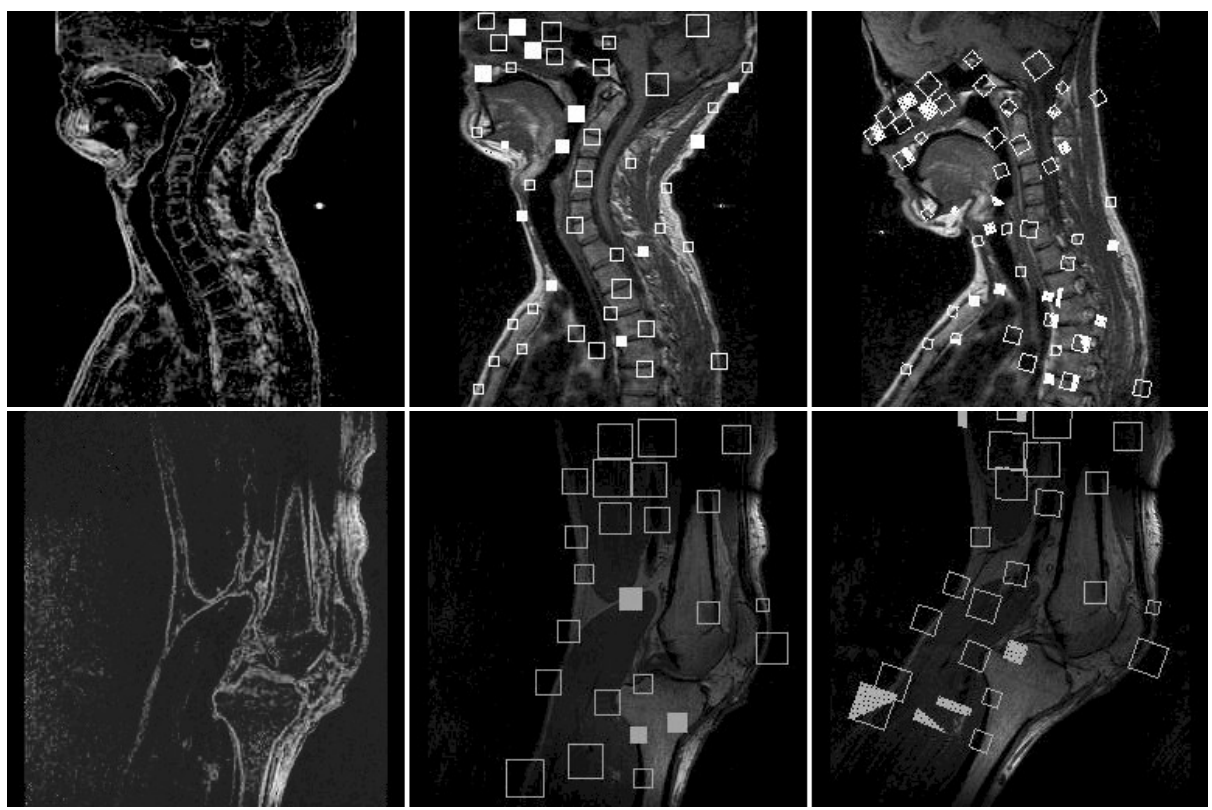

Fig. 2 Local variance distribution (left), subset of selected templates (center) and corresponding positions found by template matching (right) of the neck image (256x256x40 voxels of size 1.25x1.25x2.5 mm) and knee image pair (256x256x125 voxels, $0.98 \times 0.98 \times 1 \mathrm{~mm})$. The figure shows saggital slices of the $3 \mathrm{D}$ data sets close to the center.

Template selection addresses the following considerations:

- selection of image features that are relevant for the registration method

- determination of template size such that rigidity may be assumed within the template but that it still contains sufficient structure for the rigid registration process

- homogeneous distribution of templates within the volume of interest

An image feature that is closely related to $\mathrm{LC}$ is the local variance. At image location $\boldsymbol{x}$ local variance is defined as $\sigma_{\mathrm{L}}^{2}(\boldsymbol{x}, r)=\sum_{\boldsymbol{u} \in S(\boldsymbol{x}, r)}\left(I(\boldsymbol{u})-\bar{I}_{S}\right)^{2}$ where $S(\boldsymbol{x}, r)$ is a sphere of radius $r$ around $\boldsymbol{x}, I(\boldsymbol{u})$ is the grey value at position $\boldsymbol{u}$ and $\bar{I}_{S}$ denotes the average gray value within the sphere. The amount of local variance a template encloses serves as criterion to determine its size. If a template becomes too large for the rigidity assumption, it is rejected. The distance between template centers should be related to the scale of the deformation present in the images. Large deformations might require overlapping templates.

The idea of template propagation is to establish a chain of successfully registered templates from a starting point to the most remote template in the image. The selection of the template to be registered next as well as its starting estimates are based on the distance to previously registered templates and on the confi- 
dence in the registration result. As similarity measure for registration as well as for rating the registration result LC [10] is used. A LC value of almost one indicates a successful registration while values approaching zero correspond to poor registrations.

After all selected templates have been registered, a sub-set of template pairs is chosen as input for the elastic transformation scheme. The selection of corresponding points is performed in analogy to the template selection. Instead of a minimum amount of local variance, a minimum $\mathrm{LC}$ value is defined. This step removes misleading registration results which go along with small LC values.

\section{$3 \quad$ Experiments and Results}

The algorithm has been applied to four 3D MR image pairs, three of the human neck and one of the knee. As the results of the neck images are similar, only one neck and the knee example are reported. Image sizes and resolutions are given in the caption of fig. 2. Template selection and registration has been performed with the parameters given in tab. 1. In order to visualize the results of template selection and template matching, the outlines of template volumes are plotted into the image volume (fig. 2). Although it is possible to reveal gross registration errors by visual inspection of template positions in the reference and target image (fig. 2), a quantitative evaluation of registration accuracy requires results obtained by an independent process. For the data sets used here, pairs of corresponding points picked by an expert were available. As the landmark based deformation (fig. 4a) requires corresponding points in all image parts, the expert indicated corresponding points like "anterior thigh" in regions where no salient $3 \mathrm{D}$ anatomical points are available. In the following, the validation scheme is described and results are presented.

During template selection, templates are centered at landmark positions in the source image, even if these positions do not meet the selection criterion based on local variance. The remaining templates are selected according to section 2 and template propagation is performed. In order to investigate the accuracy of the algorithm, positions found by the expert and by template propagation have been compared by calculating the Euclidean distance $\|\Delta r\|=$ $\left\|\boldsymbol{r}_{\text {template,target }}-\boldsymbol{r}_{\text {landmark,target }}\right\|$. This procedure has been performed for the neck and knee images using 22 and 17 landmarks respectively. Results are compiled in tab. 2.

Finally, corresponding points found by template matching have been used to apply an elastic transformation incorporating rigid structures to the "neck down" image. To allow a comparison with results based on manually selected landmarks [2], information about the rigidly registered vertebra $\mathrm{C} 1-\mathrm{C} 7$ has been added and templates in related areas have been discarded. Transformed images are shown in fig. 4. 
Tab. 1 Experimental parameters. Calculation times refer to a $400 \mathrm{MHz}$ SUN UltraSPARC with $512 \mathrm{MB}$ memory. $d_{\mathrm{t}, \text { min }}$ denotes the minimum distance between template centers, $l_{\max }$ is the maximum template size.

\begin{tabular}{|l|l|c|c|c|c|c|c|}
\hline name & image & $\begin{array}{c}\text { starting } \\
\text { estimates } \\
\text { given for }\end{array}$ & $\begin{array}{c}d_{\mathrm{t}, \min } \\
\mathrm{mm}\end{array}$ & $\begin{array}{l}l_{\max } \\
\mathrm{mm}\end{array}$ & $\begin{array}{c}\text { number of } \\
\text { templates }\end{array}$ & $\begin{array}{c}\text { time for } \\
\text { selection } \\
\text { min }\end{array}$ & $\begin{array}{c}\text { time for } \\
\text { registration } \\
\text { min }\end{array}$ \\
\hline exp.1 & neck & voxel of C1 & 5 & 15 & 3879 & 6 & 31 \\
\hline exp.2 & neck & voxel of C1 & 4 & 25 & 7844 & 9 & 68 \\
\hline exp.3 & knee & patella & 10 & 29 & 564 & 4 & 28 \\
\hline exp.4 & knee & patella & 4 & 25 & 5884 & 32 & 271 \\
\hline
\end{tabular}

Tab.2 Euclidean distance between positions of corresponding points specified by an expert user and positions resulting from template matching. LC denotes the maximum local correlation value found for a template centered at the landmark position. Parameter settings for the experiments are given in tab. 1

\begin{tabular}{|c|c|c|c|c|c|c|c|c|c|}
\hline neck & $\exp$ & & exp & .2 & knee & $\exp$ & & exp & \\
\hline $\begin{array}{l}\text { landmark } \\
\text { name }\end{array}$ & $\begin{array}{c}\|\Delta r\| \\
\mathrm{mm}\end{array}$ & $\mathrm{LC}$ & $\begin{array}{c}\|\Delta r\| \\
\mathrm{mm}\end{array}$ & $\mathrm{LC}$ & $\begin{array}{l}\text { landmark } \\
\text { name }\end{array}$ & $\left|\begin{array}{c}\|\Delta r\| \\
\mathrm{mm}\end{array}\right|$ & $\mathrm{LC}$ & $\begin{array}{c}\|\Delta r\| \\
\mathrm{mm}\end{array}$ & $\mathrm{LC}$ \\
\hline Fourth ventricle & & & 1.59 & 0.34 & ttach. & & & & \\
\hline L aryter & $\overline{.39}$ & 0.48 & 1.21 & 0.73 & patella & 1.91 & 0.53 & 2.02 & 0.5 \\
\hline L carotid aor & 4.48 & 0.47 & 3.38 & 0.5 & Ant. Tibial a. & 13.89 & 0.39 & 13.1 & 0.39 \\
\hline L mandible & 1.57 & 0.53 & 3.43 & 0.49 & Anterior thigh & \begin{tabular}{|l|}
1.69 \\
\end{tabular} & 0.52 & 1.61 & 0.55 \\
\hline L orbit & 0.45 & 0.77 & 0.45 & 0.78 & Common & & & & \\
\hline L straight & 4.07 & 0.3 & & & & 1.28 & 0.32 & 1.16 & 0.36 \\
\hline L T5 rib & 1.63 & 0.59 & 1.81 & 0.59 & Gastrocnemius & 7.05 & 0.23 & 9.66 & 0.29 \\
\hline Lower sternum & 6.28 & 0.67 & 6.56 & 0.71 & Genicular a. & 8.8 & 0.29 & 2.51 & 0.54 \\
\hline Mandible & 8.36 & 0.47 & 4.7 & 0.57 & la lig. & 1.07 & 0.48 & 2.01 & 0.51 \\
\hline Pituitary & 1.74 & 0.53 & 1.99 & 0.5 & Patella tendon & 7.53 & 0.3 & 2.35 & 0.41 \\
\hline $\mathrm{R}$ arytenoid & 11.94 & 0.26 & 17.19 & 0.3 & Popliteal v. & 1.88 & 0.33 & 1.85 & 0.34 \\
\hline $\mathrm{R}$ carotid Inn & 0.99 & 0.57 & 2.25 & 0.58 & Post. calf & & & 4.77 & 0.26 \\
\hline $\mathrm{R}$ mandible & 2.04 & 0.69 & 9.69 & 0.44 & Quad. femoris & 0.75 & 0.25 & 4.64 & 0.24 \\
\hline $\mathrm{R}$ orbit & 1.13 & 0.87 & 1.21 & 0.87 & Quadriceps & & & & \\
\hline R straight sinus & 9.14 & 0.31 & 17.6 & 0.26 & fem. & 7.53 & 0.35 & 2.12 & 0.41 \\
\hline $\mathrm{R}$ T5 rib & 0.79 & 0.59 & 0.69 & 0.59 & Supf. thigh v. & 1.72 & 0.43 & 4.11 & 0.41 \\
\hline Sphenoidal sinus & 3.53 & 0.45 & 3.5 & 0.38 & . G. & & & & \\
\hline Sternum & 2.31 & 0.6 & 2.6 & 0.61 & saphenous v. & 1.57 & 0.54 & 1.57 & 0.54 \\
\hline T2 rib left & 1.58 & 0.62 & 2.18 & 0.63 & Trib.2 G. & & & & \\
\hline T2 rib right & 2.15 & 0.6 & 0.95 & 0.62 & saphenous v. & 1.51 & 0.49 & 1.42 & 0.52 \\
\hline T2 spinous process & 1.15 & 0.55 & 0.81 & 0.55 & Trib.3 g. & & & & \\
\hline Trachea bification & 5.52 & 0.38 & 5.98 & 0.38 & papHenutis v. & 1.14 & 0.58 & 1.1 & 0.65 \\
\hline
\end{tabular}




\section{Discussion}

In this section, results of template selection, template registration and the nonrigid transformation are interpreted. Fig. 2 shows that templates are positioned preferably at borders between different tissue types or at the air/tissue boundary which correspond to large values of local variance. The sizes of templates located in areas with more or less uniform gray values are considerably larger than those templates positioned on tissue borders. This is particularly visible for the knee image where the local variance of muscular tissue hardly exceeds that of the background (fig. 2).

The large amount of deformation present in the neck image is reflected in the variation of local transformation parameters. For example, the rotation angle of templates around the axis perpendicular to the plane of the slices shown in the upper part of fig. 2 varies from $+34^{\circ}$ at $\mathrm{C} 1$ to $-6^{\circ}$ at the center of the image. In the knee image pair, this rotation angle varies between $+8^{\circ}$ and $-28^{\circ}$.

It follows that the propagation of starting estimates is essential for a successful registration of small templates in these cases. Using e.g. starting estimates that are valid for the $\mathrm{C} 1$ region for templates at the center of the neck image does not result in successful registrations due to the limited capture range of the optimization procedure. Fig. 2 indicates that the propagation approach led to correct template alignment even in the lung area of the neck image where little structure is present. This visual impression is confirmed by comparing registered templates with manually picked landmarks (tab. 2). Tab. 2 shows that generally $\|\Delta \boldsymbol{r}\|$ decreases with increasing LC value as assumed before. When interpreting the contents of tab. 2 it must be taken into account that the landmarks have not been selected with respect to their suitability for template matching. In accordance with the template propagation procedure, the similarity measure can, however, be used to distinguish successfully registered templates from others. For successfully registered templates, i.e. templates with high LC values $\|\Delta \boldsymbol{r}\|$ is comparable or smaller than one voxel diameter (about $3 \mathrm{~mm}$ for the neck and about $1.7 \mathrm{~mm}$ for the knee data sets). These deviations are of the same order as typical errors in manual landmark picking. In conclusion, template propagation was successful and produced accurate results.

The landmark "lower sternum" shows a deviation of about two voxel diameters despite its LC value of almost 0.8. Fig. 3 shows enlarged views of this landmark position and its environment. In the coronal and axial slices, motion artifacts in the skin region manifest themselves as a "saw-tooth" pattern. The reason for these artifacts is most likely the regular movement of the chest due to breathing that interferes with the time interval required for the acquisition of an image slice. This explains the registration result obtained for the "lower sternum" landmark: rather than corresponding positions on the sternum, corresponding points with respect to the "saw-tooth" pattern have been detected by template matching. 

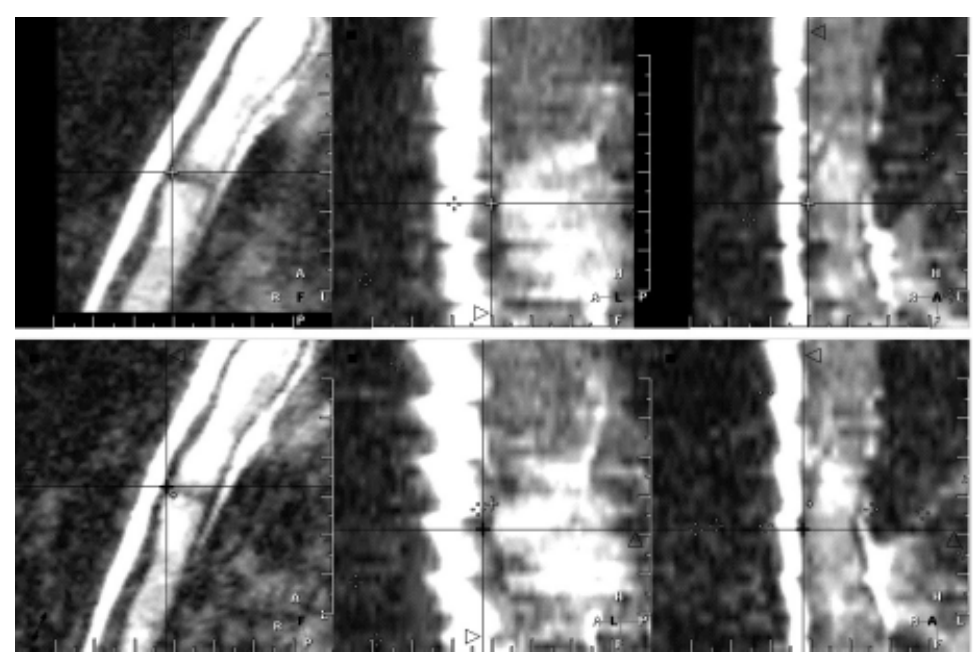

Fig. 3 Motion artifacts at the position of the "lower sternum" landmark in the "head up" image (top) and the "head down" image (bottom). From left to right, sagittal, coronal and axial slices are shown. The cross-hairs indicate corresponding positions found by template matching. Details are given in the text.

A comparison between exp.1 and exp.2 (and between exp.3 and exp.4) shows that the increase of template density and overlap increased the registration accuracy of some templates at the cost of higher computation time. The computation times between $40 \mathrm{~min}$ and $300 \mathrm{~min}$ are closely related to the number of templates and hence to the large amount of deformations considered by the algorithm.

Finally, we compare the results of two elastic transformations of the "neck down" image according to [2]. The first is based on the manually picked landmarks and the other is based on the corresponding points found by template propagation. Ideally, an elastic transformation of the "head down" image would yield the "head up" image. Thus, the performance of elastic transformation results can be evaluated by an investigation of its differences to the "head up" image. Bright areas in the difference images fig. $4 \mathrm{~b}$ and fig. $4 \mathrm{~d}$ indicate locations where misregistration has occured.

The average absolute gray value of the difference image fig. $4 \mathrm{~d}$ is by about $30 \%$ smaller than that in fig. 4b. This indicates an improvement of non-rigid registration results by using template matching rather than manually picked landmarks. The gray values in both difference images at the positions of segmented vertebra are almost zero. This follows directly from the procedure described in [2]. The improvements are particularly evident for the vertebra Th1-Th6 that have not been segmented and for the chest region. 


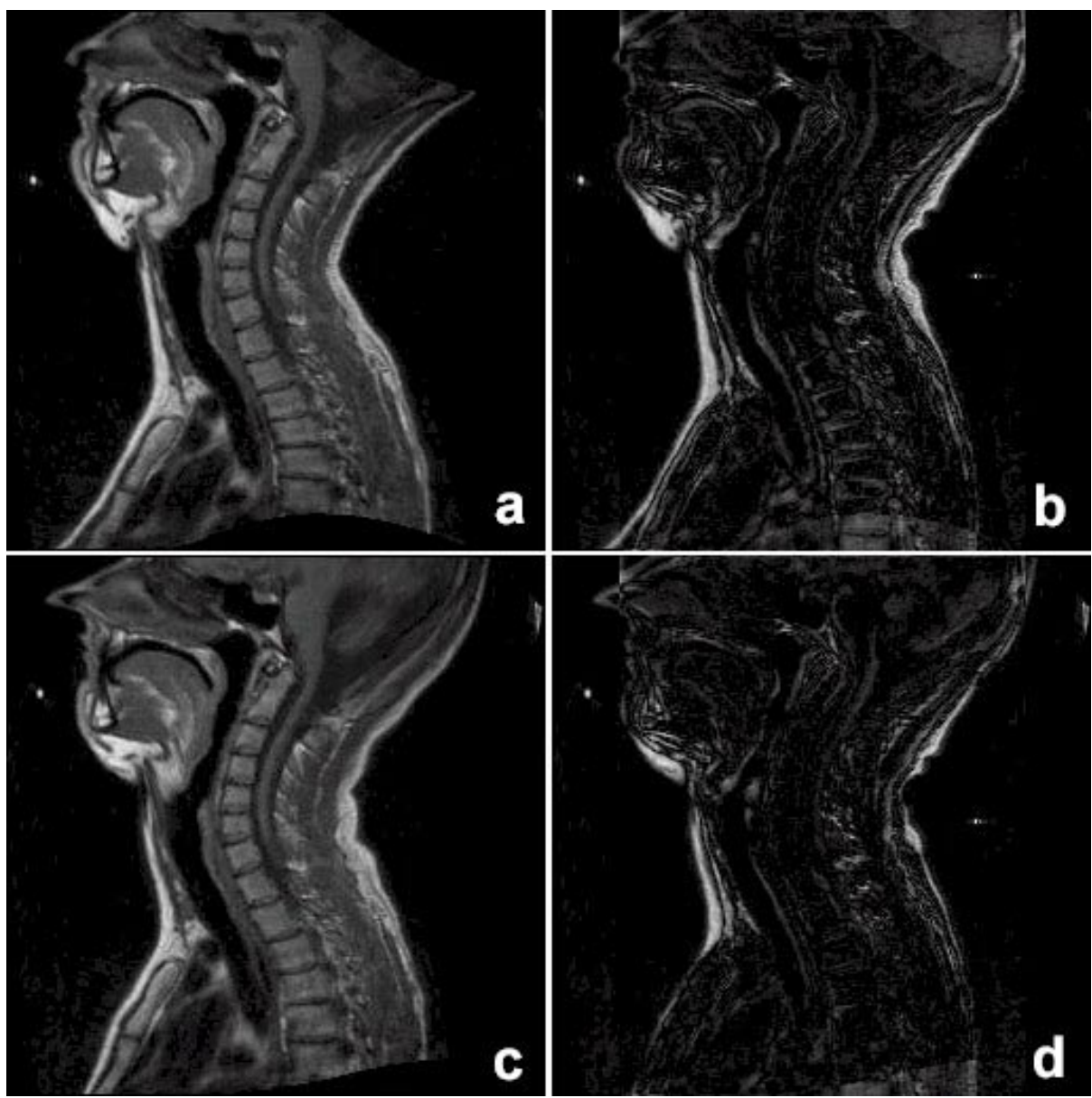

Fig. 4 Result of elastic transformations according to [2] (a,c) and difference images $(b, d)$ between transformed image and the "head up" image. The elastic transformation is based on 22 landmarks picked manually (a,b) and on 178 corresponding points determined by template matching $(\mathrm{c}, \mathrm{d})$.

It should be noted that the back of the head is only visible in the "head up" image. It follows that for several templates selected in this image, a corresponding position in the "head down" image does not exist. However, results of template registration have been used as input for deformation estimation. Although the outer shape of the occiput seems to match better in fig. 4c, closer inspection reveals the presence of artifacts due to the different field of view in both images. These artifacts could be avoided easily by restricting the volume of interest to structures present in both images. 


\section{Conclusions and Future Work}

The performance of a new automatic algorithm for robust 3D deformation field estimation has been demonstrated and tested. Given approximate rigid registration parameters at one image location only, the template propagation paradigm results in a large, evenly distributed set of homologous points between two volumes despite the presence of large deformations. These points can be the input for various elastic interpolation methods to obtain motion field estimations for an image pair. To test template propagation, deviations between corresponding points found by template registration and landmarks picked by an expert have been investigated. The average deviation for successfully registered templates are significantly smaller than one voxel diameter. Results of a registration algorithm which allows pre-segmented rigid bodies to be incorporated into a non-linear deformation have been significantly improved by using homologous points determined by template registration rather than a smaller number of manually picked landmarks. The properties of the LC measure allow for an extension to multi-modality elastic registration which will be addressed in our future work.

\section{References}

1. Bookstein, F. L.: Principal warps: Thin-plate splines and the decomposition of deformations, IEEE Trans. Patt. Anal. Mach. Intell 11 (1989) 567-585

2. Little, J. A., Hill, D. L. G., Hawkes, D. J.: Deformations Incorporating Rigid Structures. Computer Vision and Image Understanding 66 (1997) 223-232

3. Declerck, J., Subsol, G, Thirion, J.-P., Ayache, N.: Automatic retrieval of anatomical structures in 3d medical images, Lecture Notes in Computer Science 950 (1995) 153-162

4. D.Davatzikos, J.L.Prince, R.N.Bryan: Image Registration Based on Boundary Mapping, IEEE Trans. Medical Imaging 15 (1996) 112-115

5. Rabbitt, R. D., Weiss, J. A., Chreestensen, G. E., Miller, M. I.: Mapping of hyperelastic deformable templates using the finite element method, SPIE proceedings 2573 (1995) 252-265

6. Rueckert, D. Sonoda, L.I., Hayes, C., Hill, D. L. G., Leach, M. O., Hawkes, D. J.: Nonrigid Registration Using Free-Form Deformations: Application to Breast MR images, IEEE Trans Medical Imaging 18 (1999) 712-721

7. Kostelec, P. J., Weaver, J. B., Healy, D. M. Jr.: Multiresolution elastic image registration, Med. Phys. 25 (1998) 1593-1604

8. Collins, D. L., Holmes, C. J., Peters, T. M., Evans, A. C.: Automatic 3-D ModelBased Neuroanatomical Segmentation, Human Brain Mapping 3 (1995) 190-208

9. Rösch, P., Blaffert, T., Weese, J.: Multi-modality registration using local correlation, in: H. U. Lemke, M. W. Vannier, K. Inamura, A. G. Farman (Eds) CARS'99 Proceedings, Elsevier (1999) 228-232

10. Weese, J., Rösch, P., Netsch, T., Blaffert, T., Quist, M: Gray-Value Based Registration of CT and MR Images by Maximization of Local Correlation, MICCAI'99, Lecture Notes in Computer Science, 1679 (1999) 656-663

11. Studholme, C., Hill, D.L.G., Hawkes, D.J.: Automated 3-D registration of MR and CT images of the head. Med. Image Anal. 1 (1996) 163-175 